the S-RNase is necessary for SI to operate, the group went on to transform SI plants (already carrying two S-alleles) with the S-RNase associated with a third, and different, S-allele. These transformants continued to reject pollen carrying the 'endogenous' S-alleles. But they also now rejected pollen carrying the heterologous allele, demonstrating that expression of the S-RNase alone is sufficient for the stigma to reject pollen of a particular S-genotype.

Similar conclusions are drawn by Murfett et al. ${ }^{2}$. Ingeniously overcoming the problem of low expression of heterologous S-RNase in Nicotiana by driving the transgene with a strong stylar promoter, they expressed an S-RNase in a selfcompatible (SC) hybrid of Nicotiana langsdorfii and $N$. alata. The resulting transformants synthesized high levels of the heterologous S-RNase in the style, and rejected pollen from plants naturally homozygous for the S-RNase. Last year, Clarke presented data at the International Genetical Congress which, in retrospect, complement these findings. Using a Lycopersicum (tomato) line, which had mutated from SI to SC, her group showed that the 'S-RNase' polypeptide was present at normal levels, but was inactive enzymatically, the mutation having changed a key histidine residue at the RNase active site. These findings, which are not yet published, thus suggest that the enzymatic activity of the S-RNase is essential for the operation of SI.

Vital pieces of the jigsaw are still missing, by far the largest being the male factor. This molecule is presumably located on the pollen-tube surface, and must interact with the S-RNase so as to internalize it in self combinations. Further, we have no information on the features of the S-linked RNase itself which confer Sspecificity. The strong selective advantage offered by SI (ref. 7) has resulted in the evolution of a number of different nechanisms, of which RNase is only one. The study of these other SI mechanisms has, disappointingly, failed to provide any evidence of factors common to either male or female determinants. So far, we have molecular data for three types of SI system (see figure) - first, S-RNases which are found in the Solanaceae, the Scrophularaceae and the Rosaceae; second, a mechanism based on a smaller female protein, described to date only for a species of poppy, which has recently been shown to activate a calcium-mediated signalling system in the pollen tube $\mathrm{e}^{8.9}$; and third, a very different system found in the Brassicaceae, and perhaps the Asteraceae, where the 'rejection reaction' seems to be sited in the female stigmatic cells rather than the pollen.

Study of this last system has revealed yet another link between SI and hostpathogen responses, for molecular analy- sis of the S-locus in Brassica oleracea by the Nasrallahs' group has revealed the presence of sequences encoding a glycoprotein and a transmembrane serine/ threonine kinase, both of which share considerable homology for any S-allele ${ }^{10}$. Both genes seem to be expressed primarily in the female. Strikingly, the Slocus receptor kinase belongs to a newly discovered family of receptor kinases, one of which acts as a disease resistance gene in tomato 11

The search for the male determinant in SI has been complicated by an elegant early theory which predicts that male and female cells synthesize similar molecules which, in incompatible combinations, dimerize to form an active agent. Thus considerable effort continues to be expended in attempts to find S-RNases and S-glycoproteins in the pollen. Tantalizing reports of such molecules continue to appear, but unequivocal evidence has not been forthcoming. However, if many or all SI mechanisms are derived from disease-resistance systems, might the elusive pollen factor be in some way related to the 'natural' ligand of these defence mechanisms? Although this may be the case for Brassica where a receptor kinase is involved, it is unlikely to be so in the Solanaceae, where the stylar 'defence' enzymes seem not to be associated with any signalling mechanism (although the S-RNase must have acquired one at some stage). In any event, we are currently ignorant of any ligand which binds to the 'defence' kinases.

It would be wrong to paint too depressing a picture of our knowledge of SI systems for, by plant standards, they are comparatively well understood. The genetics are clearly defined, we know a good deal about the cells involved and useful molecular data are being accumulated. The results reported in this issue now provide a mechanism by which self-pollen may be rejected. Taking a more familiar analogy, we have found the spring in the mousetrap - we need next to discover the cheese and, in the fuliness of time, what makes the mouse interested in it.

Hugh Dickinson is in the Department of Plant Sciences, University of Oxford, South Parks Road, Oxford OX1 3RB, UK.

1. Lee, H.-S., Huang, S. \& Kao, T.-h. Nature 367, 560-563 (1994)

2. Murfett, J., Atherton, T. L., Mou, B., Gasser, C. S. \& McClure, B. A. Nature 367, 563-566 (1994)

3. Hodgkin, T., Lyon, G. D. \& Dickinson, H. G. New Phytol. $110.557-569$ (1988)

. Dickinson. H. G. Nature 364. 573-574 (1993).

5. Nasrallah, J. B., Kao, T.-H., Goldberg, M. L. \& Nasrallah, M. E. Nature 318, 263-267 (1985)

6. Anderson, M. A. et al. Nature 321, 38-44 (1986)

7. Whitehouse, H. L. K. Ann. Bot. 14, 198-216 (1950)

8. Foote, H. C. C. etal. Proc natn. Acad. Sci. U. S. A. (in the press).

9. Franklin-Tong V. E. Ride J.P. Read, N. D. Trewavas, A. J. \& Franklin, F. C. H. Plant J. 4. 163-177 (1993).

10. Dzelzkalns, V. A., Nasratiah, J. B. \& Nasraliah. M. E. Dev! Bioi. 153, 70-82 (1992)

11. Martin, G. B etal Science 262, 1432-1436 (1993)
DAEDALUS

\section{Elastic gas traps}

THE standard gas cylinder, says

Daedalus, is an absurdity - $50 \mathrm{~kg}$ of steel wrapped around a mere $10 \mathrm{~kg}$ of gas. He recalls that active charcoal and zeolite 'molecular sieves' can take up almost their own weight of gas in their molecule-sized pores. Sadly, only strong heat can get it out again. He is now inventing a novel elastic microporous solid that is more like a molecular sponge.

He points out that some polymers go opaque under stress (indented-letter labelling tape works this way). Tension opens innumerable microvoids in their structure, which scatter the light. Relax the stress and the voids close up; the polymer clears again. DREADCO's chemists are now taking this effect to extremes. Their goal, DREADCO's 'Gasponge', will expand under tension into an open-cell microfoam with pores well sized to accept gas molecules.

A lot of force will be needed to dilate Gasponge. Every gram will develop hundreds of square metres of internal surface, giving the polymer an energy density approaching that of TNT. It wil relax that energy by absorbing gas avidly into its internal pores, releasing the energy of absorption as latent heat. Once loaded, a Gasponge sachet will hold its charge of gas indefinitely. To get the gas out again, simply squeeze it in a vice, when the voids will close up and expel the trapped gas. Heavy pressure will be needed, and the sample will cool strongly by the uptake of latent heat.

Gasponge will hold and dispense most gases, from the hazardous chlorine and ammonia to safer ones such as carbon dioxide and butane. The one gas which presents problems is, sadly, oxygen. Like oxygen-loaded charcoal, an oxygenloaded hydrocarbon polymer would be dangerously inflammable and possibly highly explosive. For welders,

glassblowers, anaesthetists, divers and other oxygen users, Daedalus hopes to devise a special non-inflammable Gasponge based on silicone or fluorocarbon rubber.

Many other uses should open up for Gasponge. By squeezing and relaxing a sample, its latent heat flow could be exploited in a simple but powerful refrigerator. As a gas trap, it could remove exhaust fumes and foul air from enclosed spaces. A small compressible Gasponge could make a neat aerosol propellant. And an oxygenated Gasponge with a sharp melting point should expand forcefully if heated to that point. This novel smokeless non-burning explosive would be ideal for mining and tunnelling. The oxygen it released would even help the ventilation.

David Jones 